\title{
Joint Adaptive Combining and Variable Tap-Length Multiuser Detector for Underwater Acoustic Cooperative Communication
}

\author{
Zhiyong Liu ${ }^{1,2}$, Yinghua Wang ${ }^{1}$, Lizhong Song ${ }^{1}$, Yinyin Wang ${ }^{1}$ and Fusheng Dai ${ }^{1,2}$ \\ ${ }^{1}$ School of Information and Electrical Engineering, Harbin Institute of Technology \\ Weihai, 264209 - P.R. China \\ ${ }^{2}$ Science and Technology on Communication Networks Laboratory \\ Shijiazhuang, 050081 - P.R. China \\ [e-mail: lzyhit@hit.edu.cn, 952684330@qq.com, songlz@hit.edu.cn, 764151014@qq.com, dfs1963@163.com] \\ *Corresponding author: Lizhong Song and Zhiyong Liu
}

Received April 13, 2017; revised July 10, 2017; revised August 2, 2017; accepted September 17, 2017; published January 31, 2018

\begin{abstract}
In this paper, we propose a joint adaptive combining and variable tap-length multiuser detector (MUD) for amplify-and-forward (AF) underwater acoustic cooperative interleave-division multiple access (IDMA) communication system. The proposed MUD jointly realizes tap-length adjustment, adaptive combining, and multiuser detection. In contrast to the existing methods, the proposed detector can adaptively combine the received signals from different nodes at destination, and does not need the assumption that full and perfect channel state information (CSI) of all the links at the receiver is known. Moreover, the proposed detector can adaptively adjust the tap coefficient vector and tap-length of each branch according to the specific channel profile of each branch. Simulation results validate the feasibility and show the advantages of the proposed detector against existing counterparts.
\end{abstract}

Keywords: underwater acoustic cooperative communication, interleave-division multiple access, adaptive combining, variable tap-length, multiuser detector

The work was supported by Shandong Provincial Natural Science Foundation of China (ZR2016FM02), National Natural Science Foundation of China (61201145), the Graduate Education and Teaching Reform Research Project in Harbin Institute of Technology (JGYJ-201625) and the Foundation of Science and Technology on Communication Networks Laboratory. 


\section{Introduction}

Underwater acoustic communication has drawn considerable attention in both academia and industry because of the burgeoning requirement in many commercial and military applications [1]. However, underwater acoustic channel (UAC) is one of the most challenging environments for the transmission of information due to the unique characteristics of the UAC such as limited bandwidth, high and variable propagation delays, and significant scattering and multipath. The design of reliable underwater acoustic communication is a challenge.

To improve the reliablity of the links, cooperative communication is an attractive approach [2-5]. In the cooperative mode, the receiver requires to combine multiple signals received from direct path and relay paths. Therefore, the combining technique would partly influence the system performance. Most of existing studies in underwater acoustic cooperative communication(UACC) system adopt equal gain combining (EGC) [5-11] or maximum ratio combining (MRC) [5, 12-16]. The MRC approach can achieve better performance. However, the MRC assumes that all the channel state information (CSI) of all the paths is known. For practical underwater acoustic cooperative communication system, these CSIs are difficult to obtain.

In underwater acoustic cooperative communication, both intersymbol inteference (ISI) and multiple-access interference (MAI) should be considered to mitigate. Multipath delay in UAC causes severe ISI [17], which can extend over hundreds of transmitted symbols. In addition, for actual underwater acoustic communication network, multiple users are present simultaneously in both time and frequency, thus MAI has significant impact on system performance. An effective multiuser detection (MUD) strategy should be adopted to solve this problem. In order to remove MAI and ISI, chip-level adaptive decision feedback equalization based on interleave-division multiple access (DFE-IDMA) and code-division multiple access (DFE-CDMA) has been used for multiuser communication in [18]. An adaptive chip-level DFE receiver for a two-user uplink IDMA system has been studied in [19]. In both [18] and [19], fixed tap-length (FT) MUD is adopted. However, the tap-length (TL) of the FIR filter greatly influences the performance and computational complexity of the filter. With too few taps, the linear filters may not realize their full potential, in contrast, using too many taps, besides wasting computations, may increase the steady-state mean square error (MSE) [20]. For a specific underwater acoustic channel, the optimal TL to achieve optimal system performance is difficult to predict and obtain. To complicate things further, the optimum TL will change with variation of time and underwater environments, which can lead to the change of the channel profile. Therefore, the existing FT MUD cannot commendably adapt to UAC. Hence, to achieve better bit error rate (BER) performance, the MUD should be able to adaptively adjust the TL according to the specific UAC profile. To our best knowledge, there are still lack of the researches on the joint implementation of adaptive combining multiple signals and TL adjustment.

In this paper, we propose a joint adaptive combining and variable TL multiuser detector (JACVT-MUD) for underwater acoustic cooperative communication. The proposed detector can adaptively combine multiple inputs via diverse paths based on the proposed adaptive algorithm, and doesn't require to know the CSIs of all links. In addition, the proposed detector can also adaptively adjust the TL of each branch in accordance with the specific UAC profile. Hence, the proposed detector is more suitable for practical underwater acoustic cooperative communication systems. 
The rest of this paper is organized as follows. In Section II, we present the system model. In Section III, the proposed JACVT-MUD is described in detail. Section IV shows the simulation results. The conclusion is drawn in Section V.

\section{System Model}

We consider an underwater acoustic cooperative communication system with a relay node $(R)$, a destination node $(D)$ and $K$ source nodes $\left(S_{i}\right)$ as the simultaneous multiple users, $i=1,2, \cdots, K$, as depicted in Fig. 1 .

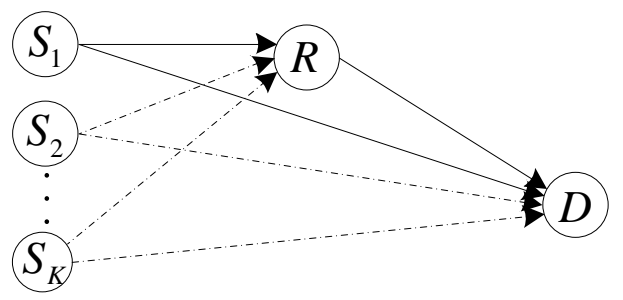

Fig. 1. Model of an underwater acoustic cooperative communication system

The source, destination, and all relays are equipped with single transmit and receive antenna. We assume the amplify-and-forward (AF) relaying, and all nodes have the same average power constraint. We also assume that interleave-division multiple access (IDMA) [21] is adopted. The transmitter structure for the source nodes is showed in Fig. 2. Let $\boldsymbol{b}_{k}=\left[b_{k}(1), b_{k}(2), \cdots, b_{k}\left(N_{b}\right)\right]^{T} \in\{0,1\}_{1}^{N_{b}}$ denote information bits of the $k$ th source node, where $b_{k}(n) \in\{0,1\}, N_{b}$ is the number of information bits. Firstly, the $\boldsymbol{b}_{k}$ are encoded by a rate $R_{c}$ scrambling repetition code, which is utilized to give the system MAI protection, generating a coded sequence

$$
\mathbf{c}_{k}=\left[c_{k}(1), c_{k}(2), \cdots, c_{k}(N)\right]^{T}
$$

where $N$ is the encoded frame length, $N=N_{b} F, F$ represents the spreading factor. Then, the coded bits are additionally interleaved by user-specific interleaver $I_{k}[\cdot]$, whose outputs are mapped to BPSK symbols

$$
\boldsymbol{x}_{k}=\left[x_{k}(1), x_{k}(2), \cdots, x_{k}(N)\right]^{T}
$$

where $N$ is the encoded frame length. Note that the spreading unit is same for all sources, and it can be equally represented as an ensemble of a bit-repetition operation and a scrambling unit. Distinct interleaver patterns are used to separate different users. 


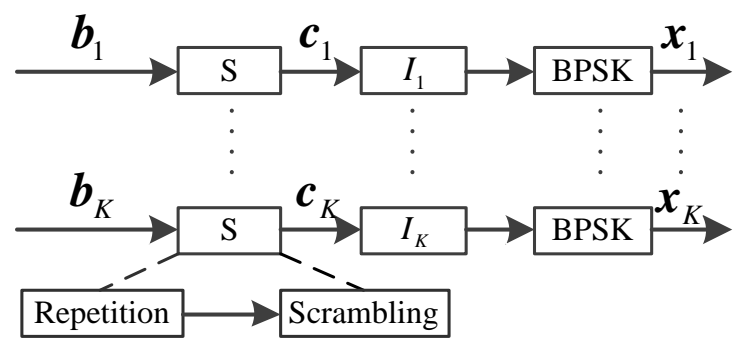

Fig. 2. Transmitter structure of IDMA

The cooperative transmission from source to destination is accomplished in two phases. Specifically, in the broadcasting phase, the source nodes transmit to the relay node and the destination node. In the relaying phase, the relay node forwards a scaled noisy version of the received signal from source to the destination node in different time slots. Without loss of generality, user 1 is designated as the expected user.

During the broadcasting phase I, the received signals at the destination and the relay terminal are given by

$$
\begin{aligned}
& r_{S_{1} D}(n)=\sqrt{P_{S_{1}}} h_{S_{1} D}(n) * x_{1}(n)+\sum_{k=2}^{K} \sqrt{P_{S_{k}}} h_{S_{k} D}(n) * x_{k}(n)+v_{S_{1} D}(n) \\
& r_{S_{1} R}(n)=\sqrt{P_{S_{1}}} h_{S_{1} R}(n) * x_{1}(n)+\sum_{k=2}^{K} \sqrt{P_{S_{k}}} h_{S_{k} R}(n) * x_{k}(n)+v_{S_{1} R}(n)
\end{aligned}
$$

where $P_{S_{k}}$ is transmitted signal power of the $k$ th source, $h_{S_{k} D}(n)$ and $h_{S_{k} R}(n)$ are underwater acoustic channel impulse responses from the $k$ th source to destination and from the $k$ th source to the relay, respectively. $h_{S_{k} D}(n)$ and $h_{S_{k} R}(n)$ can be obtained by BELLHOP model [22-23]. $v_{S_{1} D}(n)$ and $v_{S_{1} R}(n)$ reprent samples of the additive white Gaussian noise with zero mean and variance $\sigma_{i}^{2}, i \in\left\{S_{1} D, S_{1} R\right\}$.

During the relaying phase, at the destination terminal, the received signal from relay $R$ is given by

$$
r_{R D}(n)=\beta \sqrt{P_{R}} h_{R D}(n) * r_{S_{1} R}(n)+v_{R D}(n)
$$

where $\sqrt{P_{R}}$ is transmitted signal power of the relay, $h_{R D}(n)$ denotes underwater acoustic channel impulse response from relay to destination, which can also be obtained with BELLHOP model. $v_{R D}(n)$ reprents sample of the additive white Gaussian noise with zero mean and variance $\sigma_{R D}^{2}, \beta$ is the amplification factor of the relay, $\beta$ can be defined as follows

$$
\beta=\sqrt{\frac{P_{R}}{\sum_{k=1}^{K}\left|h_{S_{k} R}\right|^{2} P_{S_{k}}+\sigma_{S_{1} R}^{2}}}
$$




\section{Joint Adaptive Combining and Variable Tap-Length Multiuser Detector}

To effectively combine the outputs of multiple UACs and achieve better detection performance, the JACVT-MUD is motivated. The proposed detector structure is depicted in Fig. 3. Note the difference from the conventional VT detector (single branch detector) in point-to-point communication, (1) the proposed detector is designed for multiuser underwater acoustic communication system, both ISI and MAI need to be mitigated, (2) the number of branches of detector is greater than 1 , the output signals from multiple branches must be adaptively combined according to the corresponding channel state information of each branch. The proposed MUD will combine tap-length adjustment, combining technique, multiuser detection into a single robust receiver.

\subsection{Preparation}

In the JACVT-MUD, the tap coefficients (TC) vector of each branch is given by

$$
\boldsymbol{w}_{i}(n)=\left[w_{i, 1}(n), w_{i, 2}(n), \cdots, w_{i, m_{i}}(n)\right]^{T}
$$

where $i \in\left\{S_{1} D, R D\right\}, \boldsymbol{w}_{i}(\mathrm{n})$ is initialized with $\boldsymbol{w}_{i}(\mathrm{n})=[0,0, \cdots, 0]^{T}, m_{i}$ is the TL.

The signal vector of corresponding branch is as follows

$$
\mathbf{u}_{i}(n)=\left[r_{i}(n-1), r_{i}(n-2), \cdots, r_{i}\left(n-m_{i}\right)\right]
$$

where $i \in\left\{S_{1} D, R D\right\}$.

The output of each branch is

$$
y_{i}(n)=\boldsymbol{u}_{i}(n) \boldsymbol{w}_{i}(n)
$$

and the corresponding error is defined as

$$
e_{i}(n)=t_{i}(n)-\hat{y}_{i}(n)=\boldsymbol{u}_{i}(n) \boldsymbol{w}_{i}(n)-\boldsymbol{u}_{i}(n) \hat{\boldsymbol{w}}_{i}(n)
$$

where $t_{i}(n)$ is training sequences, $\hat{y}_{i}(n)$ is the estimate for $t_{i}(n), \hat{\boldsymbol{w}}_{i}(n)$ is the estimate of $\boldsymbol{w}_{i}(n)$.

\subsection{Proposed JACVT-MUD}

The proposed detector will directly process the sampled signals after the analogue-to-digital converter (A/D), as showed in Fig. 3. For the received signals from $S-D$ and $S-R-D$, the corresponding branch of detector will process the received signal for $r_{S_{1} D}(n)$ and $r_{R D}(n)$, respectively. At each chip bit epoch, a bit decision is made at the output of corresponding branch (SD branch and RD branch). Tap coefficients of each branch are adjusted using normalized least mean square (NLMS) algorithm. The adaptation works in training stages, initially using training sequences to adapt to the underwater acoustic channel, and then in decision directed mode using hard decisions as the output of each branch. 


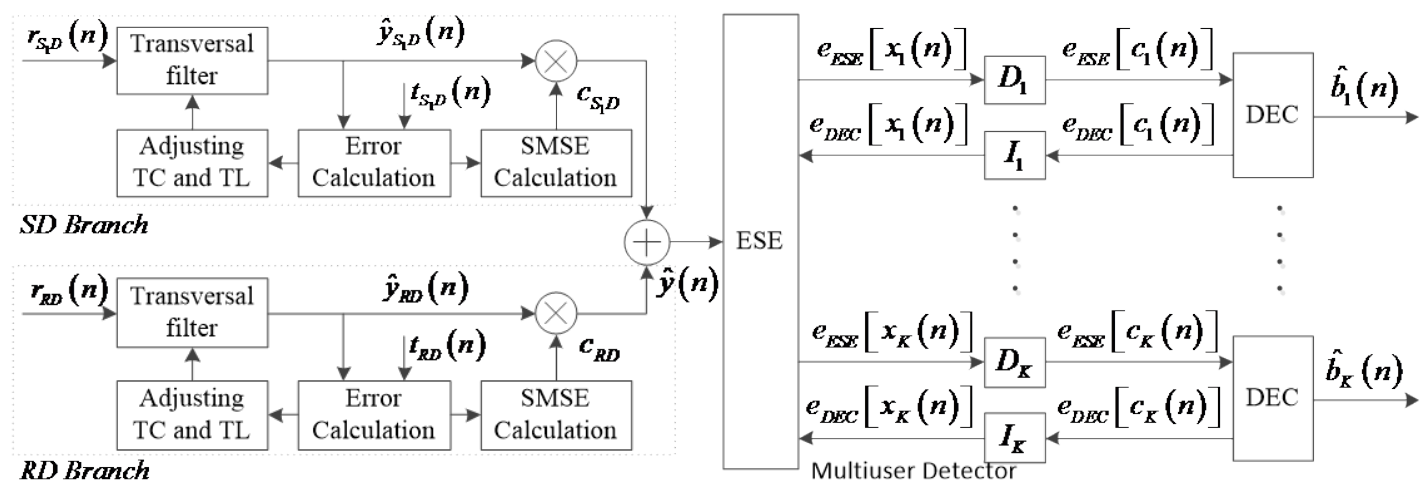

Fig. 3. The structure of JACVT-MUD

We extend the work in [20] to design the TL adjustment algorithm for each branch. The different outputs $\left(\hat{\boldsymbol{y}}_{S D}(\boldsymbol{n})\right.$ and $\left.\hat{\boldsymbol{y}}_{R D}(\boldsymbol{n})\right)$ of each branch with different TL can be used to compute a corresponding error signal $\left(\boldsymbol{e}_{n}\right)$ with (10). The distinct error signal can be squared and averaged to obtain an output mean square error (MSE) measure for corresponding branch at each tap-length.

$$
M S E_{i}(n)=E\left[\left|t_{i}(n)-\hat{y}_{i}(n)\right|^{2}\right]=\frac{\sum_{j=1}^{n} e_{i}^{2}(j)}{n}
$$

where $i \in\left\{S_{1} D, R D\right\}$.

To avoid the repetitive computation of a division, the accumulated squared error (ASE) is adopted as the measure criteria to adjust the TL of each branch, for each tap-length, ASE is defined as

$$
\operatorname{ASE}_{i}(n)=\sum_{j=1}^{n}\left|t_{i}(j)-\hat{y}_{i}(j)\right|^{2}=\sum_{j=1}^{n} e_{i}^{2}(j)
$$

where $i \in\left\{S_{1} D, R D\right\}$.

At each tap-length, the training sequences used to compute ASE is set as $N_{t}$. Ideally, each $A S E_{i}(n)$ would be smaller than the previous one

$$
A S E_{i, L}(n)<A S E_{i, L-1}(n)
$$

where

$$
\begin{gathered}
A S E_{i, L-1}(n)=\sum_{j=1}^{n} \gamma^{n-j}\left|t_{i}(j)-\hat{y}_{i, L-1}(j)\right|^{2} \\
A S E_{i, L}(n)=\sum_{j=1}^{n} \gamma^{n-j}\left|t_{i}(j)-\hat{y}_{i, L}(j)\right|^{2}
\end{gathered}
$$


where $i \in\left\{S_{1} D, R D\right\}$. In (14)-(15), $\gamma$ is a forgetting factor $(\gamma \leq 1)$, weighting the relative importance of previous samples, $y_{i, M}(j)$ is the $j^{\text {th }}$ output of the $i^{\text {th }}$ branch in the $M^{\text {th }}$ segment training sequences, and $\operatorname{ASE}_{i, M}(n)$ is the ASE of the $i^{\text {th }}$ branch obtained with the $M^{\text {th }}$ segment training sequences. The number of each segment training sequences is $N_{t}$.

The process of adjusting tap-length is as follows:

If $\operatorname{ASE}_{L}(n) \leq \alpha_{u p} A S E_{L-1}(n) \Rightarrow$ add $p$ taps

$$
\begin{gathered}
\boldsymbol{u}_{i}^{\prime}(n)=\left[r_{i}(n) r_{i}(n-2) \cdots r_{i}\left(n-m_{i}-p\right)\right] \\
\hat{\boldsymbol{w}}_{i}^{\prime}(n)=[\hat{\boldsymbol{w}}_{i}(n) \overbrace{00 \cdots 0}^{p}]^{T} \\
\hat{\boldsymbol{w}}_{i}^{\prime}(n+1)=\hat{\boldsymbol{w}}_{i}^{\prime}(n)+\frac{\mu}{\delta+\left\|\boldsymbol{u}_{i}^{\prime}(n)\right\|^{2}}\left(t_{i}(n)-\boldsymbol{u}_{i}^{\prime}(n) \hat{\boldsymbol{w}}_{i}^{\prime}(n)\right) \boldsymbol{u}_{i}^{\prime}(n)
\end{gathered}
$$

where $\alpha_{u p}$ needs to satisfy $\alpha_{u p}>0, i \in\left\{S_{1} D, R D\right\}$, both $\mu$ and $\delta$ are positive constant, $\mu$ is a step-size factor, which is used to control the change in tap coefficients vector from one iteration to the next, $\delta$ is introduced to overcome the problem of numerical calculation difficulty that may arise when $\left\|\boldsymbol{u}_{i}^{\prime}(n)\right\|^{2}$ is small.

If $\operatorname{ASE}_{L}(n) \geq \alpha_{\text {down }} A S E_{L-1}(n) \Rightarrow$ remove $p$ taps

$$
\begin{gathered}
\boldsymbol{u}_{i}^{\prime \prime}(n)=\left[r_{i}(n) r_{i}(n-2) \cdots r_{i}\left(n-m_{i}+p\right)\right] \\
\hat{\boldsymbol{w}}_{i}^{\prime \prime}(n)=\left[\hat{w}_{i, 1}(n), \hat{w}_{i, 2}(n), \cdots, \hat{w}_{i, m_{i}-p}(n)\right]^{T} \\
\hat{\boldsymbol{w}}_{i}^{\prime \prime}(n+1)=\hat{\boldsymbol{w}}_{i}^{\prime \prime}(n)+\frac{\mu}{\delta+\left\|\boldsymbol{u}_{i}^{\prime \prime}(n)\right\|^{2}}\left(t_{i}(n)-\boldsymbol{u}_{i}^{\prime \prime}(n) \hat{\boldsymbol{w}}_{i}^{\prime \prime}(n)\right) \boldsymbol{u}_{i}^{\prime \prime}(n)
\end{gathered}
$$

where $\alpha_{\text {down }}$ needs to satisfy $\alpha_{\text {down }} \leq 1, \alpha_{u p}$ and $\alpha_{\text {down }}$ should meet $\alpha_{u p} \leq \alpha_{\text {down }}$. The function of $\alpha_{u p}$ and $\alpha_{\text {down }}$ is to determine the amount of improvement or worsening necessary to force the detector to expand or contract. The closer $\alpha_{u p}$ and $\alpha_{\text {down }}$ are, the more frequently the detector will change its tap-length.

Then, after the training stage, we can obtain the steady-state MSE of each branch. The steady-state MSE of each branch is defined as

$$
\operatorname{SMSE}_{i}=E\left[\left|t_{i}(n)-\hat{y}_{i}(n)\right|^{2}\right]=\frac{\sum_{j=1}^{n} e_{j}^{2}}{n}
$$

where $i$ denotes $i^{\text {th }}$ branch, $i \in\left\{S_{1} D, R D\right\}$.

Unlike the MRC, which uses the CSIs of all links to obtain the combining coefficients, the proposed approach is based on the steady-state MSE to obtain the combining coefficients. The steady-state MSE is an important index on detection performance. The smaller the value is, the 
better performance the detector can achieve. In contrast, larger value of steady-state MSE may induce worse performance.

Therefore, the combining coefficients can be obtained according to the steady-state MSE, as follows

$$
c_{i}=\frac{1 / S M S E_{i}}{1 / S M S E_{S_{1} D}+1 / S M S E_{R D}}
$$

where $i$ denotes $i^{\text {th }}$ branch, $i \in\left\{S_{1} D, R D\right\}$. From (23), it is obvious that the smaller the steady-state MSE is, the larger the combing coefficient $c_{i}$ can be obtained. In addition, it is noted that the proposed approach does not need to know the CSIs of all links. Thus, it is more suitable for practical underwater acoustic cooperative systems.

After the combining coefficients is obtained, the outputs of all the branches can be combined to achieve diversity gain, the final output can be represented as

$$
\hat{y}(n)=c_{S_{1} D} \hat{y}_{S_{1} D}(n)+c_{R D} \hat{y}_{R D}(n)
$$

where $c_{S_{1} D}$ and $c_{R D}$ are combining coefficients for $S_{1}-D$ and $S_{1}-R-D$ paths, respectively.

At last, multiuser detection is used to separate the equalized mixed signal $\hat{y}(n)$ coming from combined output of all branches and decode the transmitted data of expected user. Multiuser detection mainly contains two parts, i.e., an elementary signal estimator (ESE) and $K$ single-user a posteriori probability (APP) decoders (DECs). Assuming that the adaptive equalization of each branch is ideal, we may represent the soft output $\hat{y}(n)$ as

$$
\hat{y}(n)=x_{k}(n)+\eta_{k}(n)
$$

where $\eta_{k}(n)=\sum_{k^{\prime}=1, k^{\prime} \neq k}^{K} x_{k^{\prime}}(n)+v(n)$ is residual distortion, consists of residual MAI and the noise signal $v(n)$. The ESE generates logarithmic likelihood ratio (LLR) which can be obtained according to [18]

$$
e_{E S E}\left[x_{k}(n)\right]=\frac{2\left\{\hat{y}(n)-E[\hat{y}(n)]+E\left[x_{k}(n)\right]\right\}}{\operatorname{Var}[\hat{y}(n)]-\operatorname{Var}\left[x_{k}(n)\right]} \quad \forall k, n
$$

where $E[\cdot]$ and $\operatorname{Var}[\cdot]$ represents the mean and the variance function, respectively. The mean and the variance of $\hat{y}(n)$ can be obtained by

$$
\begin{gathered}
E[\hat{y}(n)]=\sum_{k=1}^{K} E\left[x_{k}(n)\right], \forall n \\
\operatorname{Var}[\hat{y}(n)]=\sum_{k=1}^{K} \operatorname{Var}\left[x_{k}(n)\right]+\sigma_{v}^{2}, \forall n
\end{gathered}
$$


where $\sigma_{v}^{2}$ is the variance of total noise, the estimation for $\sigma_{v}^{2}$ can be obtained with $\hat{\sigma}_{v}^{2}=\sigma_{e}^{2}=E\left[|t(n)-\hat{y}(n)|^{2}\right]$. The mean and the variance of $x_{k}(n)$ in (26) can be calculated by

$$
\begin{gathered}
E\left[x_{k}(n)\right]=\tanh \left\{e_{D E C}\left[x_{k}(n)\right] / 2\right\}, \forall k, n \\
\operatorname{Var}\left[x_{k}(n)\right]=1-\left\{E\left[x_{k}(n)\right]\right\}^{2}, \forall k, n
\end{gathered}
$$

where $e_{D E C}\left[x_{k}(n)\right]$ generated by DEC is extrinsic logarithmic likelihood ratio (LLR) of $x_{k}(n)$, the $e_{D E C}\left[x_{k}(n)\right]$ can be calculated by

$$
e_{\mathrm{DEC}}\left[x_{k}(n)\right]=I_{k}\left[e_{\mathrm{DEC}}\left[c_{k}(n)\right]\right]-e_{\mathrm{ESE}}\left[x_{k}(n)\right]
$$

where $e_{\mathrm{DEC}}\left[c_{k}(n)\right]$ is extrinsic logarithmic likelihood ratio (LLR) of $c_{k}(n)$, which can be obtained by DEC as in [18]. In the detector, $e_{\mathrm{DEC}}\left[x_{k}(n)\right]$ is used as a priori information in ESE for the next iteration. As mentioned above, each DEC treats the output of the ESE as its input, and vice versa. During iterative operation, DECs constantly exchange LLR information with ESE. The estimation $\hat{x}_{k}(n)$ for $x_{k}(n)$ can be obtained by hard mapping with the sign function

$$
\hat{x}_{k}(n)=\operatorname{sgn}\left(e_{\mathrm{DEC}}\left[x_{k}(n)\right]\right)
$$

where $\operatorname{sgn}(\cdot)$ is the sign function. The BPSK symbols $\hat{x}_{k}(i)$ can then be despread, deinterleaved and demodulated to recover the information bits $\hat{b}_{k}(n)$.

\subsection{Complexity Analysis}

The TL is an important factor on the complexity of multiuser detector (MUD), with the TL increasing, the complexity is increased correspondingly. For variable TL (VT) MUD, in each iteration, the adjustment of TL only needs multiplication, addition and subtraction operations as shown in (12)-(21). Therefore, in one iteration, when both FT and VT MUD adopt the same TL, in addition to the same computations required by both FT and VT MUD, the increased complexity brought by the adjustment of TL is limited in VT MUD.

By further considering the fact that the proposed MUD can adjust the TL to optimal TL according to the specific underwater acoustic channel, thus, it is more suitable for practical underwater acoustic cooperative communication system.

\section{Simulation Results}

In the simulations, we consider an underwater acoustic cooperative network with two source nodes, one relay and one destination node. The quasi-static UAC is generated with Bellhop model. In the model, the sources, relay and destination node are positioned at $10 \mathrm{~m}$ from the 
sea surface, carrier frequency is set as $12 \mathrm{kHz}$, the wave height is set to $0.6 \mathrm{~m}$, the distances between source (the expected user $S_{1}$ and interference user $S_{2}$ ) and destination nodes are set as $500 \mathrm{~m}$ and $600 \mathrm{~m}$, respectively. We assume that the information bits frame length is $N_{b}=1000$, in each frame, 250 bits are used as training sequences, and the transmitter and relays have the same powers. The repetition code adopts the same spreading sequence $\{+1,-1,+1,-1,+1,-1,+1,-1\}$ for all users as in [18]. The repetition code rate $R_{c}$ is set as $1 / 8$. The transmitter uses the binary phase shift keying (BPSK) modulation. In order to separate users, random interleavers [21] are adopted. The iteration times of MUD is set as 10. For the fixed TL approach, the TLs $\left(m_{S_{1} D}\right.$ and $\left.m_{R D}\right)$ are set as 20 and 21, respectively. For the adjustable TL approach, the TL increment $p$ is 15, the initial TL is set as 9 and the number of each segment training sequences $N_{t}$ for computing ASE is set as 30 .

At first, we would obtain the optimal TL for a specific channel profile by simulation. The specific profile of UAC is realized with Bellhop model. Fig. 4 shows the effects of TL on signal-to-interference-and-noise (SINR), and the point on curve is obtained by average the SINR on each data packet. The output SINR can be calculated according to the approach in [19]. As seen from the Fig. 4, the TL is an important parameter on detection performance for the proposed detector. In this paper, considering the complexity of detector, the optimal TL is defined as the minimum TL, which can make the detector approximate the optimal SINR performance. It can be seen from Fig. 4 that the SINR is close to optimal value when TL is set between as 70-170. According to the definition of the optimal TL, we can obtain the optimal TL, which is about 70 .

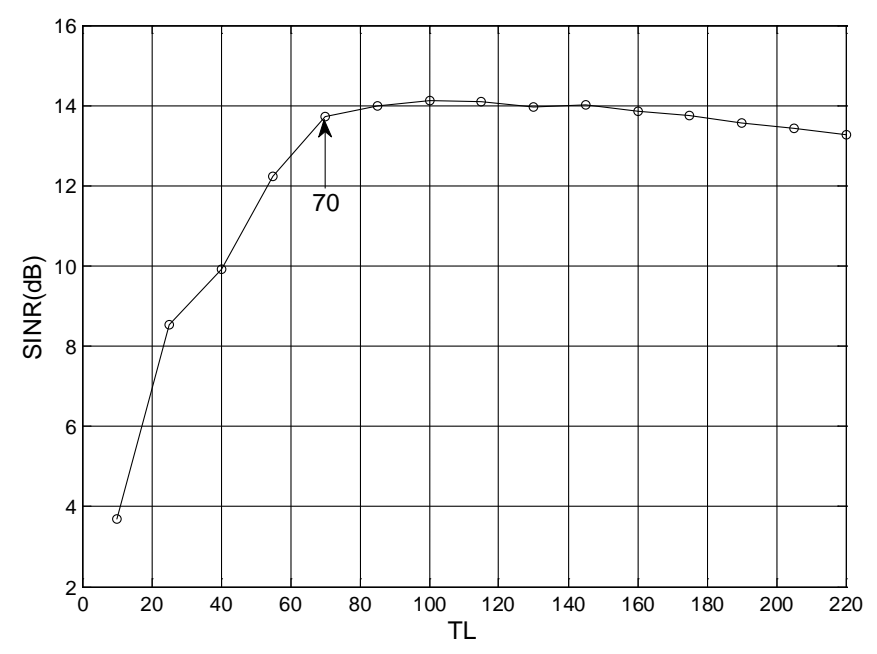

Fig. 4. The SINR performance of fixed TL detector with different TL at $S N R_{S_{1} D}=15 d B$ 


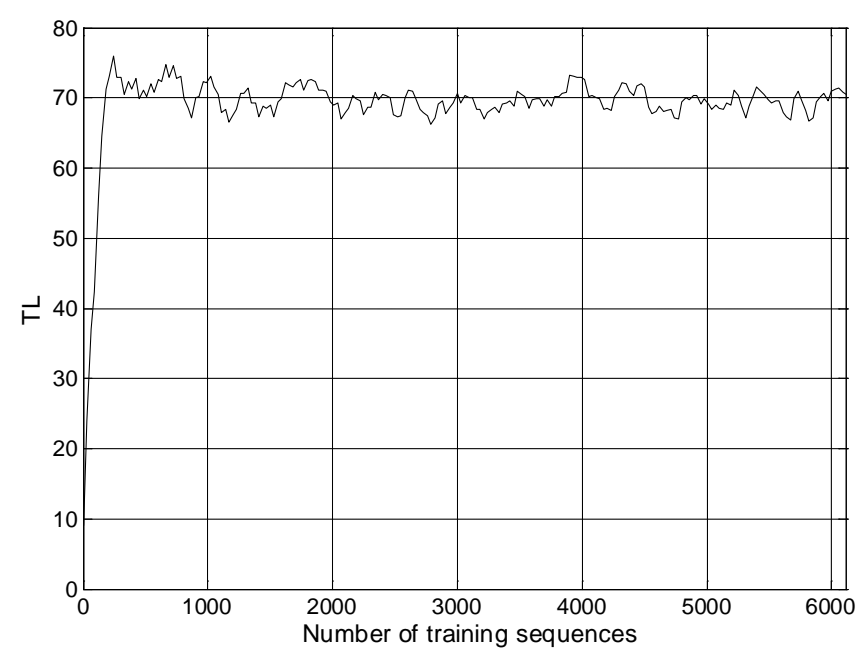

Fig. 5. TL evolution curve for VT MUD at $S N R_{S_{1} D}=15 d B$

Next, we would evaluate the ability of TL adjustment of the proposed detector. The TL evolution curve is obtained by averaging the TL evolution curve on each data packet. Fig. 5 shows the TL adjustment curve of TL. The initial TL of variable TL approach is set as 18. In (14) and (15), $\gamma$ is set to 0.999 . In (18) and (21), $\mu$ and $\delta$ are set to 0.5 and 0.6 , respectively. $\alpha_{u p}$ is set to 0.998, $\alpha_{\text {down }}$ is set to 0.999. For the same UAC used in Fig. 4, it is seen that the TL can eventually converge to the optimum TL as showed in Fig. 4. In view of the aforementioned results, it is testified that the proposed detector can adaptively adjust the TL according to the specific UAC profile.

At last, we will compare the BER and convergence performance. In order to compare the performance, a Monte-Carlo simulation is set up based on the Bellhop channel model. The BER and convergence curve performance is obtained by averaging the BER and convergence curve on 200 data packets.

In Fig. 6, we check the BER performance of different schemes, the horizontal axis denotes SNR $S N R_{S D}, S N R_{R_{1} D}=S N R_{S D}+1$. It is observed that EGC, MRC and the proposed AC scheme achieve better BER performance than the point-to-point (S-D) scheme. This is because the AC, EGC, and MRC scheme can obtain the diversity gain by cooperation of the relay node. It is also seen that the FT-MRC MUD and JACFT-MUD have evident BER performance improvement than FT-EGC scheme. This is because combining coefficients are important parameters on BER performance, both FT-MRC MUD and JACFT-MUD use better combining coefficients than EGC. In general, the BER performance of JACFT-MUD is close to that of FT-MRC MUD, however, compared with the FT-MRC MUD, the proposed JACFT-MUD scheme doesn't need to know the CSIs of all links at the receiver, and thus is more suitable for practical cooperative communication systems. In addition, it is seen that the JACVT-MUD achieves much better BER performance than JACFT-MUD. Specially, at about $\mathrm{BER}=10^{-3}$, the proposed detector performs about 8dB better than the JACFT-MUD. This is because TL is an important parameter on BER performance, the JACVT-MUD can adaptively adjust the TL of each branch according to the specific UAC profile. Fig. 7 shows the convergence performance of VT and FT MUD. It is observed that VT and FT MUD achieve close convergence rate, but VT MUD can achieve lower MSE than FT MUD. This is because 
TL is an important parameter on MSE performance, the TL can be adaptively adjusted by the VT MUD.

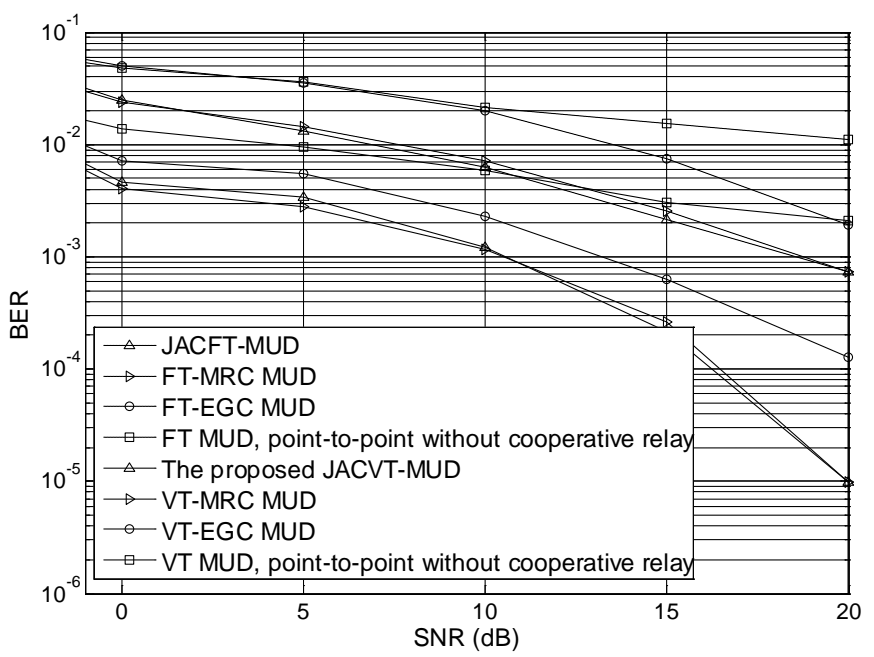

Fig. 6. BER performance comparison of the proposed detector and existing methods

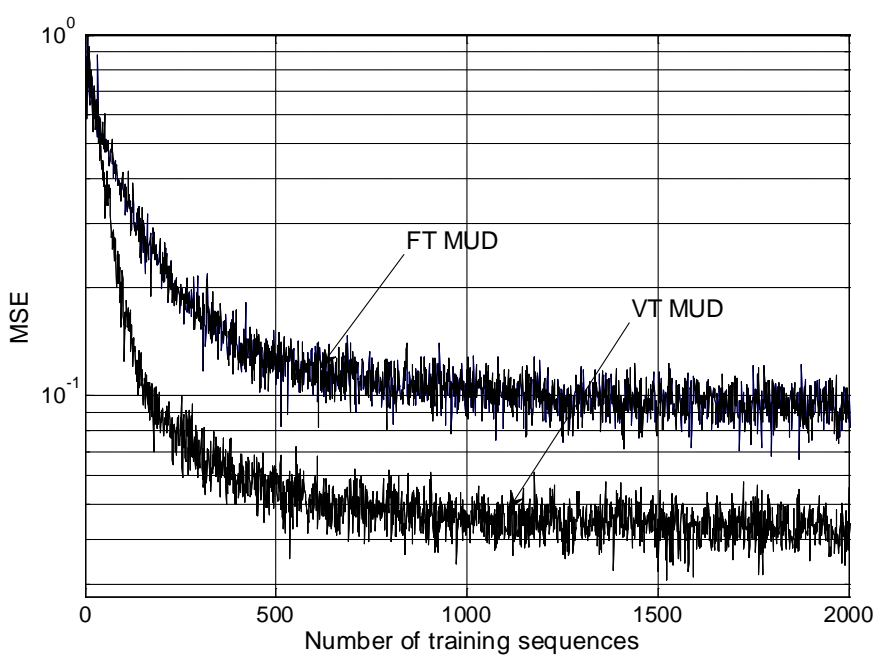

Fig. 7. Convergence performance comparison of VT and FT MUD, $S N R_{S_{1} D}=15 d B$

\section{Conclusion}

In this paper, a JACVT-MUD, which can jointly implement adaptive combining, TL adjustment of each branch according to the specific UAC profile and detection of multiuser signals, has been proposed for underwater acoustic cooperative communication IDMA system. Simulation results validate the ability of TL adjustment and the feasibility of adaptive combing. Simulation results also demonstrate that the proposed detector can achieve better performance than existing counterparts. 


\section{References}

[1] A. Khasawneh, M.S.A. Latiff, H. Chizari, M. Tariq, and A. Bamatraf, "Pressure Based Routing Protocol for Underwater Wireless Sensor Network: A Survey,” KSII Trans. Internet and Information Systems, vol. 9, no. 2, pp. 504-527, February, 2015. Article (CrossRef Link)

[2] J. Ou, H. Wu, Q. Wang and Y. Zou, "A Simple $N^{\text {th }}$ Best-Relay Selection Criterion for Opportunistic Two-Way Relay Networks under Outdated Channel State Information," KSII Trans. Internet and Information Systems, vol. 8, no. 10, pp. 3409-3422, October, 2014. Article (CrossRef Link)

[3] V. Hakami, M. Dehghan, "Distributed power control for delay optimization in energy harvesting cooperative relay networks," IEEE Transactions on Vehicular Technology, vol. 66, no. 6, pp. 4742-4755, Jun. 2017. Article (CrossRef Link)

[4] S.M. Tseng, T.L. Lee, Y.C. Ho and D.C. Tseng, "Distributed space-time block codes with embedded adaptive AAF/DAF elements and opportunistic listening for multihop power line communication networks," International Journal of Communication Systems, vol. 30, no. 1, pp. 1-12, Jan. 2017. Article (CrossRef Link)

[5] A. Saif, M. Ismail, R. Nordin and M. Fadhil, "Performance analysis of relay and combining methods in wireless networks," Journal of Theoretical \& Applied Information Technology, vol. 75, no. 1, pp. 67-80, May. 2015. Article (CrossRef Link)

[6] Y.G. Chen, Z.H. Wang, L. Wan, H. Zhou, S.L. Zhou and X.F. Xu, "OFDM -Modulated Dynamic Coded Cooperation in Underwater Acoustic Channels," IEEE Journal of Oceanic Engineering, vol. 40, no. 1, pp. 159-168, January, 2015. Article (CrossRef Link)

[7] B. Karakaya, M.O. Hasna, T.M. Duman, M. Uysal and A. Ghrayeb, "Multi- resampling Doppler compensation in cooperative underwater OFDM systems," in Proc. of 2013 MTS/IEEE OCEANS -Bergen, pp. 1-8, June, 2013. Article (CrossRef Link)

[8] S. Yerramalli and U. Mitra, "Optimal power allocation and doppler compensation in cooperative underwater networks using OFDM," in Proc. of OCEANS 2009, MTS/IEEE Biloxi-Marine Technology for Our Future: Global and Local Challenges, pp. 1-6, October, 2009. Article (CrossRef Link)

[9] S. Al-Dharrab and M. Uysal, "Outage capacity regions of multicarrier DF relaying in underwater acoustic channels," in Proc. of 2012 26th Biennial Symposium on Communications (QBSC), pp. 30-33, May, 2012. Article (CrossRef Link)

[10] M. Vajapeyam, U. Mitra, J.C. Preisig and M. Stojanovic, "Distributed space- time cooperative schemes for underwater acoustic communications," in Proc. of OCEANS 2006 - Asia Pacific, pp. 1-8, May, 2007. Article (CrossRef Link)

[11] L. Yang, R. Cao and F. Qu, "Asynchronous amplify-and-forward relay communications for underwater acoustic networks," IET Communications, vol. 10, no. 6, pp. 677-684, March, 2016. Article (CrossRef Link)

[12] Q. Song and M. Garcia, "Cooperative OFDM underwater acoustic communications with limited feedback," International Journal of Electrical, Electronics and Computer Systems (IJEECS), vol. 54, no. 16, pp. 42-46, September, 2012. Article (CrossRef Link)

[13] A.V. Babu and S. Joshy, "Maximizing the data transmission rate of a cooperative relay system in an underwater acoustic channel," International Journal of Communication Systems, vol. 25, no. 2, pp. 231-253, February, 2012. Article (CrossRef Link)

[14] X.L. Cheng, R. Cao, F.Z. Qu and L.Q. Yang, "Relay-aided cooperative underwater acoustic communications: Selective relaying," in Proc. of 2012 Oceans-Yeosu, pp. 1-7, May, 2012. Article (CrossRef Link)

[15] D.D. Tan, T.T. Le and D.S. Kim, "Distributed cooperative transmission for underwater acoustic sensor networks," in Proc. of 2013 IEEE Wireless Communications and Networking Conference Workshops (WCNCW), pp. 205-210, April, 2013. Article (CrossRef Link)

[16] C. Carbonelli, S.H. Chen and U. Mitra, "Error propagation analysis for underwater cooperative multi-hop communications,” Ad Hoc Network, vol. 7, no. 4, pp. 759-769, June, 2009. Article (CrossRef Link) 
[17] T.C. Yang, "Properties of underwater acoustic communication channels in shallow water," The Journal of the Acoustical Society of America, vol. 131, no. 1, pp. 129-145, January, 2012. Article (CrossRef Link)

[18] S.A. Aliesawi, C.C. Tsimenidis, B.S. Sharif and M. Johnston, "Iterative Multiuser Detection for Underwater Acoustic Channels,” IEEE Journal of Oceanic Engineering, vol. 36, no 4, pp. 728-744, October, 2011. Article (CrossRef Link)

[19] S.N. Qader, C.C. Tsimenidis, B.S. Sharif, M. Johnston, “Adaptive Detection for Asynchronous Uplink IDMA Shallow-Water Acoustic Channels," in Proc. of Sensor Signal Processing for Defence (SSPD 2012), pp.1-5, September, 2012. Article (CrossRef Link)

[20] F. Riera-Palou, J.M. Noras and D.G.M. Cruickshank, "Linear equalizers with dynamic and automatic length selection,” Electronics Letters, vol. 37, no. 25, pp. 1553-1554, December, 2001. Article (CrossRef Link)

[21] P. Li, L.H. Liu, K.Y. Wu and W.K. Leung, “Interleave-Division Multiple-Access,” IEEE Transactions on Wireless Communications, vol. 5, no. 4, pp. 938-947, April, 2006. Article (CrossRef Link)

[22] P. Qarabaqi, M. Stojanovic, "Statistical Characterization and Computationally Efficient Modeling of a Class of Underwater Acoustic Communication Channels," IEEE Journal of Oceanic Engineering, vol. 38, no. 4, pp. 701-717, September, 2013. Article (CrossRef Link)

[23] F.K. Jia, E. Cheng and F. Yuan, "The Study on Time-variant Characteristics of Under Water Acoustic Channels," International Conference on Systems and Informatics (ICSAI 2012), pp. 1650-1654, May, 2012. Article (CrossRef Link) 


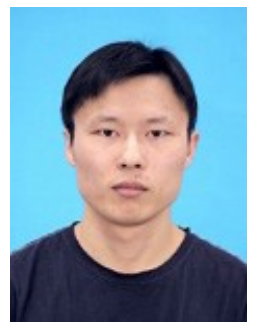

Zhiyong Liu received the Ph.D. degree in information and communication engineering from Harbin Institute of Technology Shenzhen Graduate School, Shenzhen, China, in 2010. From 2010 to 2016, he was a lecturer at the Harbin Institute of Technology (Weihai), Weihai, China, where he is currently a associate professor. His research interests include signal processing for wireless communications, cooperative communication, and underwater acoustic communication.

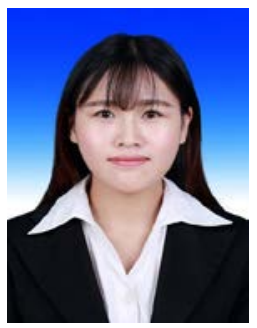

Yinghua Wang received the B.E. degree in information and communication engineering from Anhui University of Science and Technology, Huainan, China, in 2015. She is currently studying in School of Information and Electrical Engineering at Harbin Institute of Technology (Weihai) for a M.E. degree. Her research interests include cooperative communication, underwater acoustic communication, and multiuser detection.

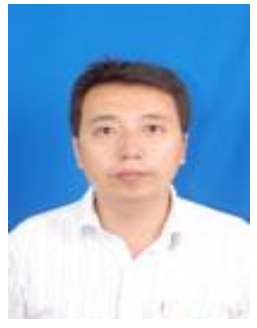

Lizhong Song received the Ph.D. degree in information and communication engineering from Harbin Institute of Technology, Harbin, China, in 2005. From 2001 to 2012, he was a lecturer, associate professor at the Harbin Institute of Technology (Weihai), Weihai, China, where he is currently a professor. His research interests include wireless communication and network, antenna design, wireless electromagnetic wave propagation, microwave technology and radar signal processing.

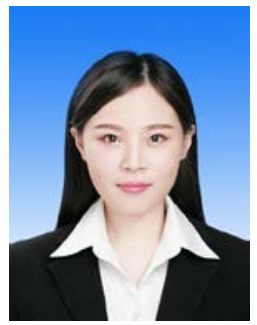

Yinyin Wang received the B.E. degree in information and communication engineering from Anhui University of Science and Technology, Huainan, China, in 2016. She is currently studying in School of Information and Electrical Engineering at Harbin Institute of Technology (Weihai) for a M.E. degree. Her research interests include underwater acoustic communication, adaptive modulation and coding.

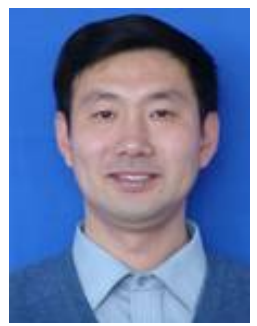

Fusheng Dai received the M.E. degree in information and communication engineering from the 54th Research Institute of China Electronics Technology Group Corporation, Shijiazhuang, China, in 1991. From 1991 to 1995, he was a lecturer, associate professor at the Harbin Institute of Technology (Weihai), Weihai, China, where he is currently a professor. His research interests include wireless communications and networks, signal processing. 